\title{
Fournier's Gangrene: Experience with Management of 46 Cases in a Tertiary Institution
}

\author{
Sani Ali Aji", Sani Usman Alhassan, Musa Muhammad Ujudud \\ Urology Unit, Department of Surgery, Aminu Kano Teaching Hospital, Kano, Nigeria \\ Email: "saniaji2004@yahoo.com
}

Received April 25, 2012; revised May 30, 2012; accepted June 7, 2012

\begin{abstract}
Background: Fournier's gangrene is a rapidly spreading necrotizing gangrene affecting the perineum, Perianal and genital regions but remarkably sparing the testicles, bladder and rectum due to their separate blood supply which is directly from the aorta. The aim of this study is to share our experience with the management of 46 cases. Patients and method: We retrospectively analysed the medical records of 46 patients admitted with Fournier's gangrene between April 2005 and December 2011 in the urology unit of Aminu Kano Teaching Hospital. Data extracted from these include age, sex, hospital stay, premorbid diseases, mobility, mortality, laboratory investigations and treatments carried out. Results: Fourty six patients were admitted and managed for Fournier's gangrene during the study period. All the patients were male and mean age was 50 years (range 20 - 80 years). Five patients died (10.90\%) mortality, $41(89.10 \%)$ patients survived. The shortest hospital stay was 13 days and longest was 120 days. $45.7 \%$ of the patients had urethral stricture with watering can perineum as predisposing factor, $16(34.80 \%)$ had diabetes mellitus, $7(15.2 \%)$ had perineal injuries as the predisposing factors while $5(10.90 \%)$ patients had uraemia and one patient $(2.20 \%)$ each had bladder tumour and scrotal abscess as their predisposing factors, in 3 patients $(6.50 \%)$ it was idiopathic. Only $34(73.90 \%)$ patients had wound swab microscopy culture and sensitivity on admission. The culture grew Klebsiella spp. in 9 (26.50\%), Staph aureus and E. coli in $5(10.90 \%)$ of cases each, while Gram positive rod and Gram negative Cocci with $4(11.80 \%)$ and $6(17.60 \%)$ respectively. Conclusion: Fournier's gangrene which is a rapidly progressive, fulminant polymicrobial synergistic infection of the perineum and genitals, is now changing pattern. Extensive surgical debridement and broad spectrum intravenous antibiotics remain the mainstay of treatment.
\end{abstract}

Keywords: Fournier's Gangrene; Management; Changing Pattern

\section{Introduction}

Fournier's gangrene is a rapidly spreading necrotizing gangrene affecting the perineum, perianal and genital regions but remarkably sparing the testicles, bladder and rectum due to their separate blood supply which is directly from the aorta. The testis may be affected if there is specific testicular pathology such as epididymorchitis or from a retroperitoneal infection spreading along the spermatic fascia causing testicular artery thrombosis [1]. It is a polymicrobial synergistic infection caused the aerobic, anaerobic, gram positive and negative bacteria, yeast and sometimes mycobacteria [2,3]. It can progress to a fulminant soft tissue infection rapidly spreading along the fascial planes, causing necrosis of the skin, subcutaneous tissue and fascia and consequent septicemia. The local spread of gangrene is at the rate of $2-3 \mathrm{~cm}$ per hour. It is named after a Parisian venereologist Jean-Alfred Fournier who first discovered it in 1883.

${ }^{*}$ Corresponding author.
It cuts across the international boundaries with a rising incidence, due to increasing ageing population, prevalence of diabetes mellitus, immunosuppressive therapy and multi-resistant pathogens. Although thought to be uncommon, it usually present as surgical emergency with high morbidity and prolonged hospital stay with a huge financial burden on the patient and sometimes the relatives, however the mortality rate is decreasing.

FG commonly affects men but does not spare women who constitute about 10\% (Kilic et al. 2001; Quatan and Kirby 2004) and also afflicts children. It occurs commonly among those in their $5^{\text {th }}$ and $6^{\text {th }}$ decade. Many terms were used to describe it including necrotizing cellulitis, periurethral phlegmon, phagedena, streptococcal scrotal gangrene and idiopathic scrotal gangrene [4].

Predisposing factors include diabetes mellitus, local trauma, urine leakage, perirectal or perineal surgery, extension of periurethral, anal infection, anorectal abscess, genitourinary infection, alcoholism, Immunosuppression and renal or hepatic disease [5]. 
The most commonly cultured organisms are Escherichia coli, Bacteroides, beta-hemolytic Streptococci, Staphylococcus spp. and Proteus spp., others are Fusobacterium and Clostridium perfringens.

Aggressive teamwork is the key to the successful treatment of these patients with complex problems. The use of a multidisciplinary approach using the expertise of the urologist, the reconstructive surgeon, and a general surgeon as an operative team is critical to the management.

The aim of our study is to report our experience with the management of Fournier's gangrene.

\section{Patients and Methods}

All patients who were diagnosed and managed for Fournier's gangrene in Aminu Kano Teaching Hospital, North Central Nigeria between April 2005 and December 2011 were included in this study. Their medical records were retrospectively studied noting the age, sex, predisposing factors, duration of hospital stay and outcome of treatment. Also noted were the results of Complete Blood Count and wound swab $\mathrm{m} / \mathrm{c} / \mathrm{s}$. The Diagnosis was made from history and physical examination. The data collected was analyzed using SPSS version 12 .

\section{Results}

A total of fourty six patients were admitted and managed for Fournier's gangrene during the study period. All the patients were male and mean age was 50 years, range 20 80 years (Figure 1). Five patients died (10.90\%) mortality, $41(89.10 \%)$ patients survived. The shortest hospital stay was 13 days and longest was 120 days (Figure 2). $27(65.85 \%)$ patients had low PCV (less than 28\%) on admission, $10(24.39 \%)$ patients had normal PCV while $4(9.76 \%)$ patient did not have PCV done, 9 (21.95\%) patients had deranged serum $\mathrm{U}, \mathrm{E}$ and $\mathrm{Cr}$, while 26 (63.41\%) patients had normal U, E and $\mathrm{Cr}$ and 6 (14.64\%) patients did not have it done.

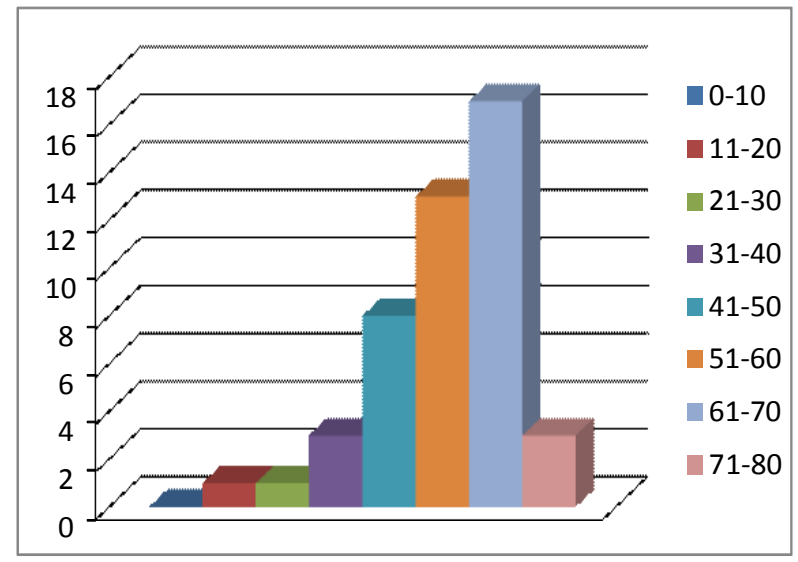

Figure 1. Distribution by age of the patients (years).

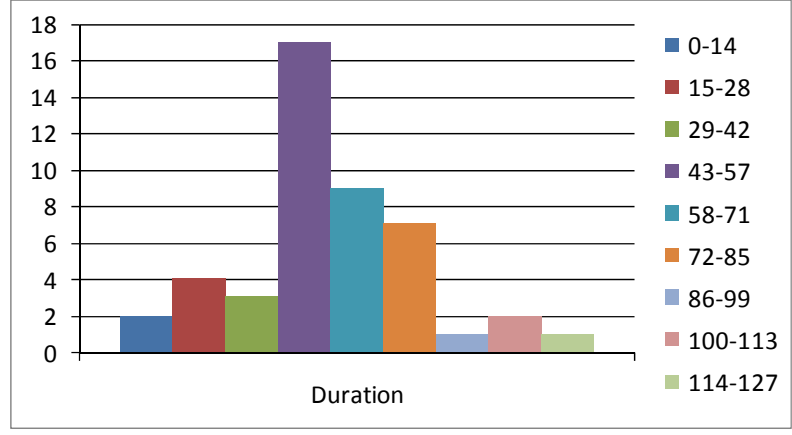

Figure 2. Duration of hospital stay (days).

$45.7 \%$ of the patients had urethral stricture with watering can perineum as predisposing factor, 16 (34.80\%) had diabetes mellitus, $7(15.2 \%)$ had perineal injuries as the predisposing factors while $5(10.90 \%)$ patients had uremia and one patient $(2.20 \%)$ each had bladder tumour and scrotal abscess as their predisposing factors, in 3 patients $(6.50 \%)$ it was idiopathic (Table 1).

All the 46 patients had a course of broad spectrum intravenous antibiotics with serial debridement and sitz bath with hypertonic saline. 21 (45.70\%) patients had suprapubic cystostomy for diverting urine. One patient $(2.20 \%)$ had orchidectomy due to gangrenous testes.

Only 34 (73.90\%) patients had wound swab microscopy culture and sensitivity on admission. The culture grew Klebsiella spp. in $9(26.50 \%)$, Staph aureus and E. coli in $5(10.90 \%)$ of cases each, while Gram positive rod and Gram negative Cocci with 4 (11.80\%) and 6 (17.60\%) respectively.

The sensitivity pattern showed $24 \%$ sensitive to gentamicin, $17 \%$ each sensitive to ceftriazone and ciprofloxacin while $12 \%$ were sensitive to augmenting, chloramphenicol and tetracycline, with only $6 \%$ sensitive to ofloxacin (Figure 3).

Figures 4 and 5 showed one of the patient with Fournier's gangrene before and after debridement respectively.

Reconstruction was done in $5(10.87 \%)$ patients who had split thickness skin graft, while $3(6.50 \%)$ patients had secondary wound closure with the skin undermined.

\section{Discussion}

Fournier's gangrene which was initially characterize as being rare and idiopathic and affecting males only is now changing pattern. 46 patients were managed in our centre during the study period with increasing incidence, as 18 cases were seen in the year 2011 (Figure 6) as compared to 2005 and 2006 where one and 3 cases were seen respectively. This increasing incidence might be attributeable to rising incidence of diabetes mellitus and urethral stricture in our environment. The mean age of the pa- 
Table 1. Predisposing factors.

\begin{tabular}{ccc}
\hline Predisposing Factors & No. of Patients & Percentage (\%) \\
\hline Urethral Stricture & 21 & 45.70 \\
Diabetes Mellitus & 16 & 34.80 \\
Perineal Injury & 7 & 15.2 \\
Scrotal Abscess & 1 & 2.20 \\
Uraemia & 5 & 10.90 \\
Bladder Tumour & 1 & 2.20 \\
Idiopathic & 3 & 6.50 \\
\hline
\end{tabular}
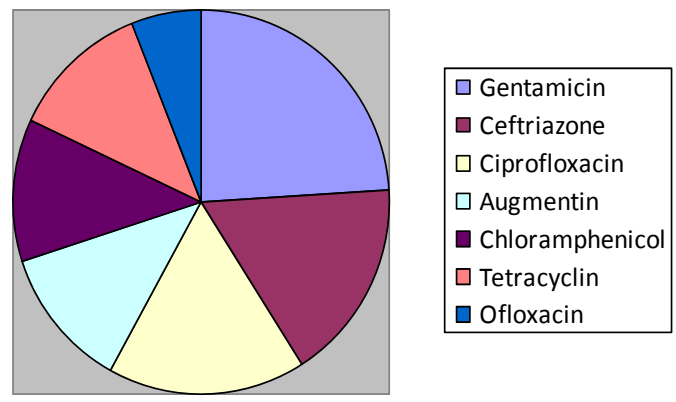

Figure 3. Sensitivity pattern.

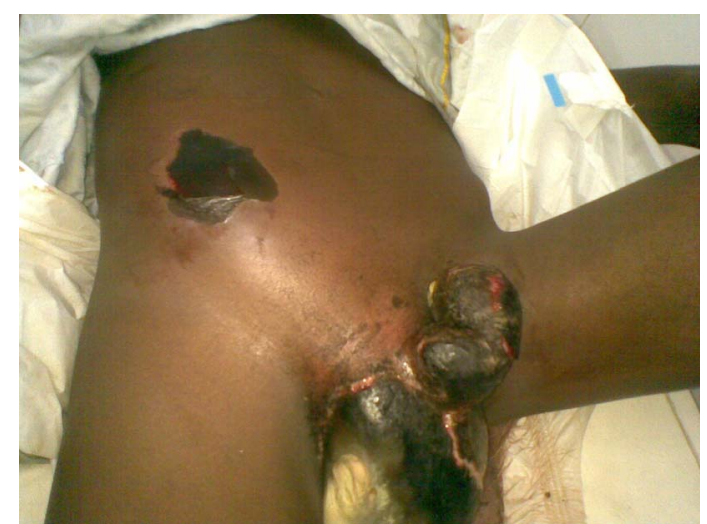

Figure 4. Fournier's gangrene in a 17 years old boy with stricture involving the perineum and anterior abdominal wall.

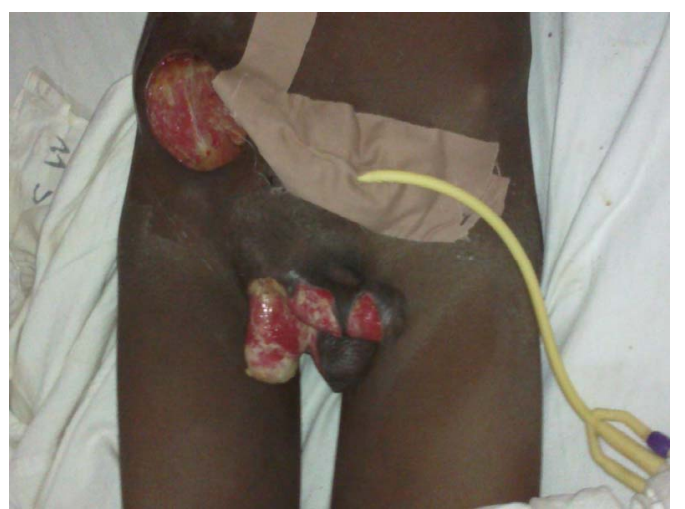

Figure 5. Same patient after debridement, SPC and sitz bath.

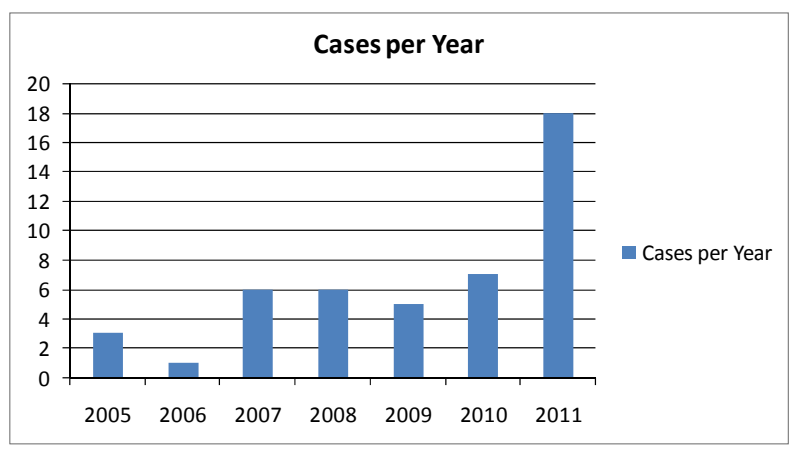

Figure 6. Distribution of case Fournier's gangrene per year.

tients was 50 years with a range of $20-80$ years this is similar to studies done elsewhere [6-10]. Although it is also known to affect children and females, none of our patients is below 20 years and no female was found.

Only $6.5 \%$ of the patients in our study were found not to have any predisposing factor. This is in accordance with studies done by Arshad et al. 2008 and Ikramullah et al. 2009. Out of the 43 patients with known predisposing factors, Urethral stricture with watering can perineum and perineal abscess is on the lead (45.7\%). Most studies found diabetes mellitus as the leading predisposing factor [11-13] this is not different from our study were $34.8 \%$ of the patients where diabetic.

The main stays of treatment of Fournier's gangrene are resuscitation, broad spectrum intravenous antibiotics and radical debridement [14-16], same principles were followed in our centre. In addition to above other centers in the world use hyperbaric oxygen $[17,18]$ but in our centre we do not have such facility instead all the patients had twice daily hypertonic saline sitz bath after the initial debridement with a very good response. All the patients with urethral stricture $(45.7 \%)$ had urinary diversion in form of suprapubic cystostomy. It is known from the literature that the testicles were rarely involved in the gangrene process since they have different blood supply [19-21] from our study only one patient had orchidectomy from gangrenous testis, this is one of the patients that had scrotal abscess probably from infected hydrocele.

Mortality from Fournier's gangrene is generally high, 4 to $67 \%[12,16,20]$. This is due to the rapid progression of the disease associated with sepsis and presence of comorbid illnesses. The mortality from our study was $10 \%$ which is not different from the findings in other centers.

\section{Conclusion}

Fournier's gangrene which is a rapidly progressive, fulminant polymicrobial synergistic infection of the perineum and genitals, is now changing pattern. Extensive surgical debridement and broad spectrum intravenous 
antibiotics remain the mainstay of treatment in order to reduce the morbidity and mortality.

\section{REFERENCES}

[1] M. Hohenfellner and R. A. Santucci, "Emergencies in Urology," Springer, Berlin, 2007, pp. 50-59. doi:10.1007/978-3-540-48605-3

[2] D. K. Rajan and K. A. Scharer, "Radiology of Fournier's Gangrene," AJR, Vol. 170, No. 1, 1998, pp. 163-168.

[3] R. N. Uppot, H. M. Levy and P. H. Patel, "Fournier Gangrene: Role of Imaging," RadioGraphics, Vol. 28, 2008, pp. 519-528. doi:10.1148/rg.282075048

[4] A. Thwaini, A. Khan, A. Malik, et al., "Fournier's Gangrene and Its Emergency Management," Postgraduate Medical Journal, Vol. 82, No. 970, 2006, pp. 516-519. doi:10.1136/pgmj.2005.042069

[5] S. C. Ndirika, R. Melville and J. Green, "Fournier's Gangrene in a Man Who Was HIV-Positive with a High CD4 Count: An Unusual Presentation of a Complex Rectoscrotal Fistula," UroToday International Journal, Vol. 3, No. 5, 2010, p. 12.

[6] W. M. Ghnnam, "Fournier's Gangrene in Mansoura Egypt, a Review of 74 Cases," Journal of Postgraduate Medicine, Vol. 54, No. 2, 2008, pp. 106-109.

[7] M. Ulug, E. Gedik, S. Girgin, et al., "The Evaluation of Microbiology and Fournier's Gangrene Severity Index in 27 Patients," International Journal of Infectious Diseases, Vol. 13, No. 6, 2009, pp. e424-e430. doi:10.1016/j.ijid.2009.01.021

[8] A. Antonio, D. C. N. Filho and L. M. Montovani, "Management of Fournier's Gangrene: Experience of a University Hospital of Curitiba," The Journal of the Brazilian College of Surgeons, Vol. 37, No. 6, 2010, pp. 435-441.

[9] I. Khan, "Experience in Management of Fournier's Gangrene: A Review of 19 Cases," Gomal Journal of Medical Sciences, Vol. 7, No. 1, 2009, pp. 65-67.

[10] A. M. Malik, S. Sheikh, R. Pathan, et al., "The Spectrum of Presentation and Management of Fournier's Gangrene -An Experience of 73 Cases," Journal of the Pakistan
Medical Association, Vol. 60, No. 8, 2010, pp. 617-619.

[11] J. Medina-Polo, et al., "Fournier's Gangrene: Evaluation of Prognostic Factors in 90 Patients," Actas Urológicas Españolas, Vol. 32, No. 10, 2008, pp. 1024-1030.

[12] A. Hanan, F. Hajji and T. O. Ismail, "Hyperbaric Oxygen Therapy Additional Debridement in the Control of Fournier's Gangrene, the Utility of the Score of a Severity Index in Predicting the Severity of the Disease and Patient Survival," Actas Urológicas Españolas, Vol. 35, No. 6. 2011, pp. 332-338. doi:10.1016/j.acuroe.2011.01.007

[13] R. S. Hollabaugh Jr., et al., "Fournier's Gangrene: Therapeutic Impact of Hyperbaric Oxygen," Plastic and Reconstructive Surgery, Vol. 101, No. 1, 1998, pp. 94-100. doi:10.1097/00006534-199801000-00016

[14] B. Unal, et al., "Fournier's Gangrene Approaches to Diagnosis and Treatment," Saudi Medical Journal, Vol. 27, No. 7, 2006, pp. 1038-1043.

[15] M. Grabe, T. E. Bjerklund-Johansen, H. Botto, et al., "Guidelines on Urologic Infections," European Association of Urology Guidelines, 2011.

[16] Z. T. Stockinger, "Fournier's Gangrene Case Report," Naval Medical Center, 2004.

[17] M. Ulug, et al., "The Evaluation of Microbiology and Fournier's Gangrene Severity Index in 27 Patients," International Journal of Infectious Diseases, Vol. 13, No. 6, 2009, pp. e424-e430. doi:10.1016/j.ijid.2009.01.021

[18] R. B. Levenson, et al., "Fournier Gangrene: Role of Imaging," The Journal of Continuing Medical Education in Radiology, Vol. 28, No. 2, 2007, pp. 519-528.

[19] N. Eke, "Fournier's Gangrene: A Review of 1726 Cases," British Journal of Surgery, Vol. 87, No. 6, 2000, pp. 718728. doi:10.1046/j.1365-2168.2000.01497.x

[20] K. Korhonen, "Hyperbaric Oxygen Therapy in Acute Necrotizing Infections with a Special Reference to the Effects on Tissue Gas Tensions," Annales Chirurgiae et Gynaecologiae, No. 214, 2000, pp. 7-36.

[21] P. R. Carrol, et al., "Necrotizing Soft-Tissue Infections of the Perineum and Genitalia Etiology and Early Reconstruction," The Western Journal of Medicine, Vol. 144, No. 2, 1986, pp. 174-178. 